\title{
Study on Effect of Simvastatin on Regeneration of Hippocampal Dentate Gyrus Nerve after Cerebral Ischemia in Rats
}

\author{
Z. MA, NA GUO, H. MA1, XUENA MA ${ }^{1}$, Y. XU AND L. GENG* \\ Department of Critical Care Medicine, The Fourth Central Hospital of Baoding City, Baoding City, 072350, Hebei province, \\ ${ }^{1}$ Department of Neurology, The Fourth Central Hospital of Baoding City, Baoding City, 072350, Hebei province, China
}

\begin{abstract}
Ma et al.: Rehabilitation of patients with coronary heart disease after percutaneous coronary intervention
This study is to explore the effect of simvastatin on regeneration of hippocampal dentate gyrus nerve after cerebral ischemia in rats. Morris water maze test is an important experimental method which has been established to judge the learning and memory of animals in the last twenty years. Except for the sham operation group, animals in the other two groups show neurologic impairment of varying degrees after waking, characterized by turning to the affected side, toppling, being unable to stand, and being unable to walk. The number of bromodeoxyuridine positive cells in simvastatin group is significantly larger than that in middle cerebral artery occlusion group, indicating that statins may play a role in promoting nerve regeneration, alleviating brain tissue damage and improving prognosis after cerebral ischemia. We haven't made the mechanism of simvastatin for promoting nerve regeneration clear-cut, and some scholars believe that simvastatin may regulate neural cell apoptosis by influencing cell signaling pathway.
\end{abstract}

Key words: Simvastatin, cerebral ischemia in rats, hippocampal dentate gyrus, nerve according to the traditional view, the brain tissue lacks self-repairing capacity after

According to the traditional view, the brain tissue lacks self-repairing capacity after damage. However, studies in recent years have shown that the brain tissue has neural stem cells (NSCs) which have many differentiation potentials and continuously divide and proliferate. These stem cells are mainly located around the lateral ventricle and Hippocampal dentate gyrus $(\mathrm{HDG})^{[1]}$. Although the brain tissue can conduct selfrepair, the purpose of repairing damage can't be achieved because of the small number of new nerve cells. Therefore, it is necessary to find some interventions to enhance endogenous nerve regeneration after damage and reduce neonatal cell death ${ }^{[2]}$. Simvastatin is a kind of lipid-lowering drug. In recent years, studies have found that simvastatin can also promote nerve regeneration and protect neurological function ${ }^{[3]}$. Hippocampus is closely related to learning and memory function and is often involved in cerebral ischemia ${ }^{[4]}$. In the experiment, the rat model of permanent middle cerebral artery occlusion (MCAO) is established by suture method to simulate acute ischemic brain damage in human. Simvastatin is given after ischemia. The changes of regeneration of HDG nerve after ischemia and the ability of learning and memory in rats are observed $^{[5]}$. The results show that simvastatin can promote the regeneration of HDG nerve cells and improve the function of learning and memory after cerebral ischemia ${ }^{[6]}$. Ischemic cerebrovascular disease (ICVD) is a disease that seriously impairs human health and has a high mortality rate and disability rate ${ }^{[8]}$. The main pathological mechanism is ischemic hypoxic damage of brain nerve cells caused by cerebral blood circulation disorder ${ }^{[7]}$. At present, the treatment of ICVD mainly includes thrombolysis, antiplatelet aggregation, anticoagulation, neuroprotection, and rehabilitation exercise. The purpose of ultra-early thrombolytic therapy is to reduce neuron damage, restore blood perfusion in infarct area and save ischemic penumbra. Thrombolytic methods include intravenous thrombolytic therapy and arterial thrombolytic therapy. The commonly used thrombolytic agents are streptokinase (SK), urokinase (UK) and tissue-type plasminogen activator (TPA ${ }^{[9]}$. Generally, the time window of intravenous thrombolysis is considered to be within $3 \mathrm{~h}$ of onset. According to the "Guidelines for Prevention and Treatment of Cerebrovascular Disease in China", the patients with cerebral infarction within 6 h of onset can be treated with intra-arterial thrombolytic 
therapy in units with experience and conditions. At present, only a few patients with ICVD can be treated by thrombolytic therapy due to the limitation of treatment time window and medical condition. Therefore, how to effectively promote the recovery of neurological function in patients with cerebral ischemia has become a research hotspot in the treatment of $\mathrm{ICVD}^{[9]}$. Simvastatin is a kind of widely used statins, which plays a significant role in lowering blood lipid. Recent studies have shown that simvastatin also has the effects of anti-atherosclerosis, anti-inflammation, protection of cardio-cerebral vessels, protection of nerves, and promotion of nerve regeneration in addition to lowering blood lipid. A large number of studies have verified that preventive use of statins can effectively reduce the incidence rate of ICVD and alleviate ischemic brain damage. However, there are few studies on whether simvastatin can promote nerve regeneration and protect neurological function after cerebral ischemia. This study adopts morris water maze (MWM) test, which is an important experimental method to judge the learning and memory of animals established in recent $20 \mathrm{y}$. In recent years, it has become the common research means of neuropharmacology, neurophysiology, neuropsychology, and gerontology. The experimental animals are provided by an animal experimental center of the Department of Basic Medicine of a university. Healthy adult male clean Wistar rats aging 3-4 mo and weighing $250 \mathrm{~g}-300 \mathrm{~g}$, are fed at $20^{\circ}-24^{\circ}$ with normal and free drinking. During the course of the experiment, rats are fed and observed in cages in animal rooms of the Department of Basic Medicine. All the operation and feed of experimental animals are in accordance with the management and feeding regulations of experimental animals, and follow the principle of humanity. A total of 90 healthy male Wistar rats are randomly divided into 3 groups. Group A: sham operation group, 30 rats. Group B: MCAO group, 30 rats. Group C: simvastatin group, 30 rats (MCAO+simvastatin). Each group is divided into 5 subgroups according to 5 time points $(3 \mathrm{~d}, 7 \mathrm{~d}, 14 \mathrm{~d}$, $21 \mathrm{~d}$ and $28 \mathrm{~d}$ ) after operation with 6 rats in each subgroup. Nylon fishing line of $0.26 \mathrm{~mm}$ diameter is selected and is cut into several sections and each section is $22 \mathrm{~mm}$ long. It is blunted with sandpaper at the head end to prevent damage to blood vessels. Mark the points that are $6 \mathrm{~mm}, 12 \mathrm{~mm}$ and $18 \mathrm{~mm}$ from the head end and disinfect it with alcohol, and then put it in normal saline. Rats are anesthetized by intraperitoneal injection with $10 \%$ of water and chlorine $(0.32 \mathrm{ml} / 100 \mathrm{~g})$. After the anesthesia takes effect, the animals are supine and fixed on the operating table. The neck is disinfected with iodophor, and the right common carotid artery (CCA) triangle is exposed by median incision. The right CCA, internal carotid artery (ICA) and external carotid artery (ECA) are isolated under an operating microscope. The right CCA near the heart is ligated and the ECA is ligated. The artery between ECA and ICA is connected by electrocoagulation and ICA is threaded for standby use. At the upper end of CCA ligation, cut a small hole near the bifurcation of CCA and insert ICA along CCA with prepared fishing line, and ligate ICA when the specified length (about $18 \mathrm{~mm}$ ) is reached. The insertion depth of nylon thread is about $18 \mathrm{~mm}$ with its head end at the beginning of MCA, which blocks the blood flow of MCA. (Don't insert in the pterygopalatine artery). Suture the incision. In sham operation group, only blood vessels are separated and blood flow isn't blocked. The five-grade marking system is adopted. 0: no nerve injury symptom; 1: unable to fully extend the contralateral front claw; 2: walking towards the contralateral side; 3 : unable to stand and fall to the contralateral side; 4 : unable to walk spontaneously and lose consciousness. 1-3 marks mean that the model is successful while 0 and 4 marks mean that the model is failing. The experimental animals are provided by an animal experimental center of the Department of Basic Medicine of a university. Healthy adult male clean Wistar rats aging 3-4 months and weighing $250 \mathrm{~g}-300 \mathrm{~g}$, are fed at $20^{\circ}-24^{\circ}$ with normal and free drinking. During the course of the experiment, rats are fed and observed in cages in animal rooms of the Department of Basic Medicine. Feed is provided by the animal center of the Department of Basic Medicine. All the operation and feed of experimental animals are in accordance with the management and feeding regulations of experimental animals, and follow the principle of humanity. A total of 90 healthy male Wistar rats are divided into 3 groups: sham operation group, MCAO group and simvastatin group. In sham operation group, rats' are skins are only incised to separate subcutaneous tissue and blood vessels, and blood flow isn't blocked. Each group is divided into subgroups by 5 time points according to the time of ischemia ( $3 \mathrm{~d}$, $7 \mathrm{~d}, 14 \mathrm{~d}, 21 \mathrm{~d}$ and $28 \mathrm{~d}$ ) with 6 rats in each subgroup. Nylon fishing line of $0.26 \mathrm{~mm}$ diameter is selected and is cut into several sections and each section is $22 \mathrm{~mm}$ long. It is blunted with sandpaper at the head end to prevent damage to blood vessels. Mark the points that are $6 \mathrm{~mm}, 12 \mathrm{~mm}$ and $18 \mathrm{~mm}$ from the head end and disinfect it with alcohol, and then put it in normal saline. Rats are anesthetized by intraperitoneal injection with 
$10 \%$ of water and chlorine $(0.32 \mathrm{ml} / 100 \mathrm{~g})$. After the anesthesia takes effect, the animals are supine and fixed on the operating table. The neck is disinfected with iodophor, and CCA triangle is exposed by median incision. The right CCA, ICA and external ECA are isolated under an operating microscope. The right CCA near the heart is ligated and the ECA is ligated. The artery between ECA and ICA is connected by electrocoagulation and ICA is threaded for standby use. At the upper end of CCA ligation, cut a small hole near the bifurcation of CCA and insert ICA along CCA with prepared fishing line, and ligate ICA when the specified length (about $18 \mathrm{~mm}$ ) is reached. The insertion depth of nylon thread is about $18 \mathrm{~mm}$ with its head end at the beginning of MCA, which blocks the blood flow of MCA. (Don't insert in the pterygopalatine artery). Suture the incision. In sham operation group, only blood vessels are separated and blood flow isn't blocked. The five-grade marking system is adopted. 0 : no nerve injury symptom; 1 : unable to fully extend the contralateral front claw; 2: walking towards the contralateral side; 3: unable to stand and fall to the contralateral side; 4: unable to walk spontaneously and lose consciousness. 1-3 marks mean that the model is successful while 0 and 4 marks mean that the model is failing. Except for the sham operation group, animals in the other two groups show neurologic impairment of varying degrees after waking, characterized by turning to the affected side, toppling, being unable to stand, and being unable to walk. The specific data are shown in Table 1.This study is to observe the nerve regeneration in the recovery period of cerebral ischemia, so the $28^{\text {th }}$ $\mathrm{d}$ is chosen as the observation time point after ischemia to observe the change of learning and memory ability of rats in each group. The results are as follows: after training, rats have learned to visit the maze. The memory function of rats in MCAO group decreases and the escape latency prolongs after $28 \mathrm{~d}$. The difference between MCAO group and sham operation group is statistically significant $(\mathrm{p}<0.05)$. Compared with that in MCAO group at the same time, the escape latency in simvastatin group is shorter. The difference is statistically significant $(\mathrm{p}<0.05)$, as shown in Table 2 . At the time point of $3 \mathrm{~d}$ after cerebral ischemia, Bromodeoxyuridine (BrdU) positive cells in hippocampal dentate gyrus of sham operation group, MCAO group and simvastatin group appear in varying degrees and there are brown particles which mainly distribute in nucleus. In sham operation group, BrdU positive cells are scattered with small round nucleus. The BrdU positive cells increase significantly in the other 2 groups. The BrdU positive cells begin to increase $3 \mathrm{~d}$ after ischemia and reach a peak on the $14^{\text {th }} \mathrm{d}$ with a high level. The BrdU positive cells begin to decrease on the $21^{\text {st }} \mathrm{d}$ and decrease sharply on the $28^{\text {th }} \mathrm{d}$. Compared with that in MCAO group, the number of positive cells in simvastatin group increases significantly $(\mathrm{p}<0.05)$ with statistical significance, as shown in Table 3 and fig. 1-3. The results show that the number of BrdU positive cells in simvastatin group is significantly larger than that in MCAO group, suggesting that statins might play a role in promoting nerve regeneration, alleviating brain tissue damage and improving prognosis after cerebral ischemia. We haven't made the mechanism of simvastatin for

TABLE 1: SCORES OF NERVE FUNCTION DEFECT IN ANIMALS

\begin{tabular}{lcc}
\hline Group & N & Score \\
\hline Sham operation group & 30 & 0 \\
MCAO group & 30 & $2.68 \pm 0.76$ \\
DMSim & 30 & $2.96 \pm 0.64$ \\
\hline
\end{tabular}

TABLE 2: WATER MAZE RESULTS OF 28D TIME POINT RATS IN EACH GROUP

\begin{tabular}{lc}
\hline Group & Achievement \\
\hline Sham operation group & $14.45 \pm 2.35$ \\
MCAO group & $79.67 \pm 4.25$ \\
DMSim & $38.35 \pm 3.47$ \\
\hline
\end{tabular}

\begin{tabular}{|c|c|c|c|}
\hline $\begin{array}{l}\text { Point of } \\
\text { time }\end{array}$ & $\begin{array}{c}\text { Sham operation } \\
\text { group }\end{array}$ & MCAO group & DMSim \\
\hline $3 d$ & $3.61 \pm 1.62$ & $9.36 \pm 3.26$ & $17.96 \pm 1.24$ \\
\hline $7 d$ & $3.78 \pm 2.07$ & $18.49 \pm 2.83$ & $32.24 \pm 2.35$ \\
\hline $14 d$ & $3.29 \pm 1.46$ & $38.24 \pm 4.28$ & $58.25 \pm 1.59$ \\
\hline $21 d$ & $2.82 \pm 1.24$ & $33.60 \pm 1.63$ & $40.25 \pm 2.21$ \\
\hline $28 \mathrm{~d}$ & $3.55 \pm 1.52$ & $16.28 \pm 3.07$ & $28.32 \pm 3.57$ \\
\hline
\end{tabular}

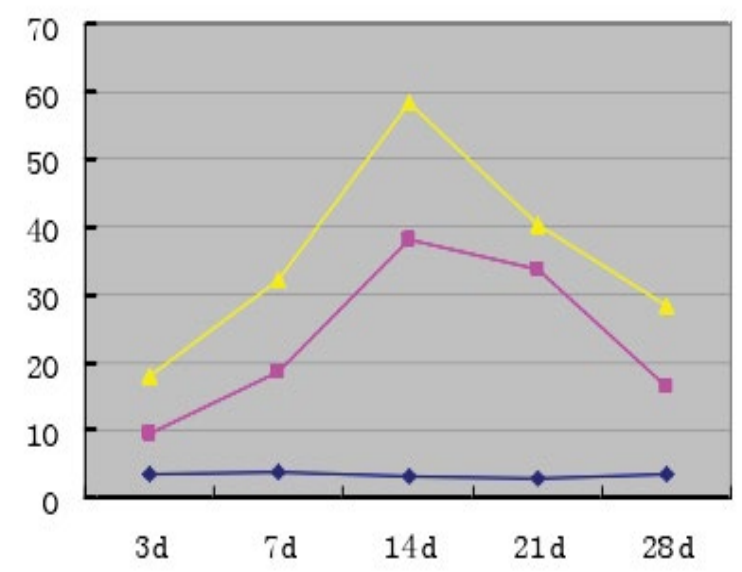

Fig. 1: Expression of DG BrdU immunoreactive cells in hippocampus of rats in each experimental group 


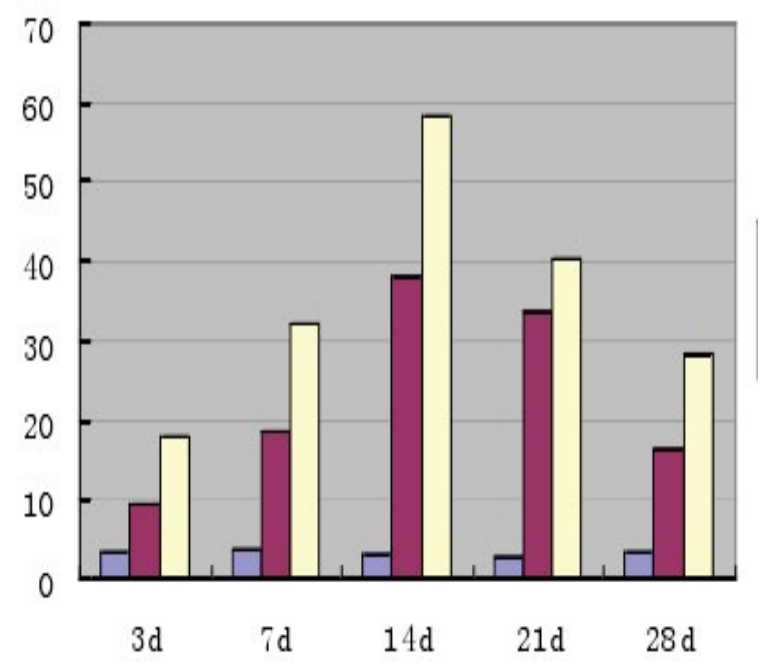

Fig. 2: Comparison of DG Brd $U$ immunoreactive cells in hippocampus of rats in each experimental group

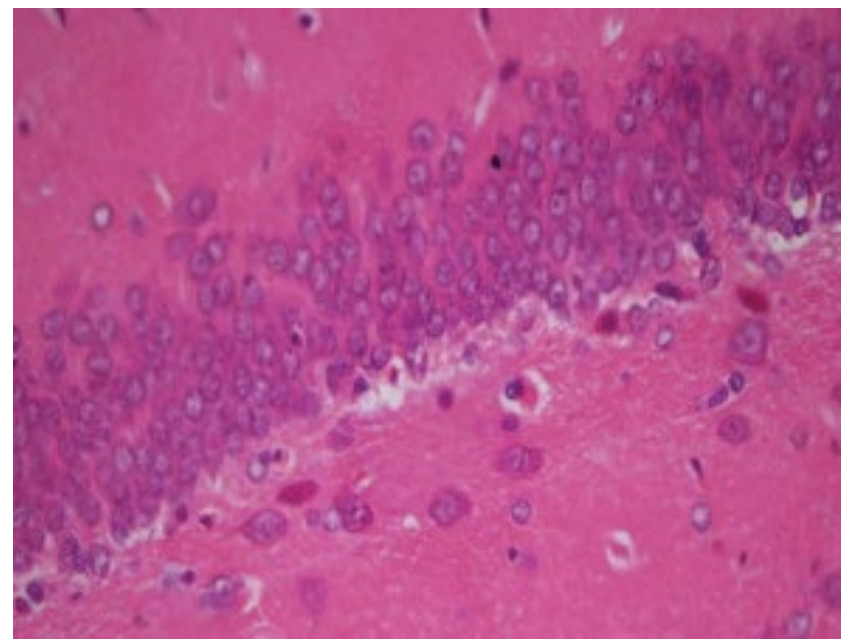

Fig. 3: Morphology and structure of 3D HE 400 cells in the sham operation group were normal.

promoting nerve regeneration clear-cut, and some scholars believe that simvastatin may regulate neural cell apoptosis by influencing cell signaling pathway.

\section{Conflict of Interests:}

The authors declared no conflict of interest.

\section{REFERENCES}

1. Monje ML, Toda H, Palmer TD. Inflammatory Blockade Restores Adult Hippocampal Neurogenesis. Science 2003;302(5651):1760-5.

2. Schinder AF, Gage FH. A hypothesis about the role of adult neurogenesis in hippocampal function. Physiol 2004;19(5):253.

3. Pistikova A, Brozka H, Stuchlik A. Adult neurogenesis in the hippocampus from a perspective of discrimination and generalization: a hypothesis. Physiol Res 2017;66(3):441.

4. Mcavoy K, Besnard A, Sahay A. Adult hippocampal neurogenesis and pattern separation in DG: a role for feedback inhibition in modulating sparseness to govern populationbased coding. Front Syst Neurosci 2015;9(120):1-7.

5. Martino G, Pluchino S. The therapeutic potential of neural stem cells. Nat Rev Neurosci 2006;7(5):395-406.

6. Ng SY, Semple BD, Morgantikossmann MC, Bye N. Attenuation of microglial activation with minocycline is not associated with changes in neurogenesis after focal traumatic brain injury in adult mice. J Neurotrauma 2012;29(7):1410-25.

7. Mcgowan P, Dougherty $H$. storm as freed tony martin sells story for six-figure sum; deal with tabloid challenges guidelines on payments to convicted criminals. Curr Pharm Des 2014;20(26):84-98.

8. Mckee M, Raine R. Riots on the streets. Bmj2011;343(7):d5248.

9. Castillo M. Checkpoint in Montebello: Inciting Riots, Up against the Wall and Earning the Right to be on the Street with Signs that Say Retén. Lat Stud 2008;6(1-2):212-215.

10. Shoesmith D. Timor-Leste: Divided Leadership in a SemiPresidential System. Asian Surv 2003;43(2):231-252.

This is an open access article distributed under the terms of the Creative Commons Attribution-NonCommercial-ShareAlike 3.0 License, which allows others to remix, tweak, and build upon the work non-commercially, as long as the author is credited and the new creations are licensed under the identical terms

This article was originally published in Special
issue on "Trends in therapeutic Management of
Various Conditions" Indian J Pharm Sci 2020:82(3)
spl issue 8;68-71

